\title{
Programa de comportamiento organizacional en la satisfacción laboral en los docentes de la Carrera Profesional de Contabilidad de la Facultad de Ciencias Económicas, Administrativas y Contables de la Universidad Nacional de Ucayali - Pucallpa, 2019
}

\section{Program of organizational behavior in job satisfaction in teachers of the Professional Accounting Career of the Faculty of Economic Administrative and Accountants Sciences of the National University of Ucayali - Pucallpa, 2019}

\author{
Rolando Carlos Canteño Gavino ${ }^{1}$ y Wilmer Ortega Chavez ${ }^{2}$
}

\begin{abstract}
${ }^{1}$ Unversidad Nacional de Ucayali. Carretera Federico Basadre, Km 6. Pucallpa, Perú. Correo electrónico: rolando_canteno@unu.edu.pe. ORCID: https;//orcid.org/0000-0003-2238-6269

${ }^{2}$ Universidad Nacional Intercultural de la Amazonía Peruana. Carretera a San José $0.63 \mathrm{Km}$. Pucallpa, Perú. Correo electrónico: wortega1978@ gmail.com. ORCID: https://orcid.org/0000-0002-5888-2902
\end{abstract}

\section{Resumen}

El estudio responde a una investigación experimental sobre el Programa de comportamiento organizacional en la satisfacción laboral en los docentes de la Carrera Profesional de Contabilidad de la Facultad de Ciencias Económicas, Administrativas y Contables de la Universidad Nacional de Ucayali - Pucallpa, 2019” en el cual su propósito fue analizar de qué manera el programa del comportamiento organizacional permite la satisfacción laboral, donde las precisiones específicas responde a las dimensiones formuladas como problemas específicos; así mismo se precisa que el tipo de investigación fue experimental con el diseño pre experimental. Con una población de 18 docentes y como muestra fueron los mismos, entonces denominado población muestral que fueron estudiada, donde los instrumentos aplicados fueron distribuidos de acuerdo a las dimensiones de las variables. Como resultado se creó una base de datos obtenidos del cuestionario $\mathrm{N}^{\circ} 1$ y 2 , estos fueron procesados a nivel descriptivo, además se contrastó la hipótesis con la prueba de $t$ de student mediante SPSS. Los resultados indicaron que el Programa de comportamiento organizacional influye significativamente en la satisfacción laboral de los docentes de la Carrera Profesional de Contabilidad de la Facultad de Ciencias Económicas y Administrativas de la Universidad Nacional de Ucayali-Pucallpa, 2019; entendiéndose como un alta significativa en la mayoría de las dimensiones fueron los mismos que demostraron que hay un alta significativa.

Palabras clave: Programa, carrera, contabilidad, laboral, satisfacción, organización, comportamiento.

\section{Abstract}

The study responds to an experimental investigation entitled Program of organizational behavior in job satisfaction in teachers of the Professional Career of Accounting of the Faculty of Economic Administrative and Accountants Sciences of the National University of Ucayali-Pucallpa, 2019 in which it was its purpose of Analyze how the organizational behavior program allows job satisfaction, where specific details respond to the dimensions formulated as specific problems; Likewise, it is specified that the type of research was experimental with the pre-experimental design With a population of 18 teachers and as a sample they were the same then called the sample population that were studied, where the instruments applied were distributed according to the dimensions of the 
variables. As a result, a database obtained from questionnaire No. 01.02 was created, these were processed at a descriptive level, in addition the hypothesis was tested with the student's t-test using SPSS. The results indicate that the Organizational Behavior Program significantly influences the job satisfaction of teachers of the Professional Accounting Career of the Faculty of Economic Administrative and Accountants Sciences of the National University of Ucayali-Pucallpa, 2019 being understood as a significant high in the majority of the dimensions are the same that show there is a significant high.

Keywords: Program, career, accounting, labor, satisfaction, organization, behavior.

\section{Introducción}

El estudio abarcó como mejorar la satisfacción laboral a través de la implementación y aplicación del programa comportamiento organizacional en los docentes de la carrera profesional de Contabilidad de la Universidad Nacional de Ucayali, por ende, hoy en día muchas instituciones del sector público buscan ser reconocidas como organizaciones atractivas donde la gente quiera trabajar. Algunas de las instituciones han empezado a buscar ser parte de premiaciones como las otorgadas por "Arellano Marketing" que premia a las empresas más atractivas para laborar, con lo que se permiten atraer a la mayor cantidad de personal capacitado. (Arellano, 2017). Sin embargo hay una cosa muy importante comprender la satisfacción laboral entendida como el resultado de factores motivadores donde se analizan factores del cargo que ocupa el trabajador y esta está en función del contenido o de las actividades desafiantes y estimulantes que conlleva como el interés que el trabajador tiene sobre el trabajo, la responsabilidad sobre sus labores el reconocimiento que siente sobre sus actividades laborales, la realización personal y el avance y crecimiento sobre su desarrollo profesional. Herzberg (2011). Se entiende por el comportamiento organizacional como una manifestación objetiva de la estructura de la organización el cual se forma porque los miembros están expuestos a las características estructurales comunes de una organización (Quispe, 2012). Así mismo, Palma (2004) sostiene que la cultura organizacional en el ámbito laboral es entendida como la percepción sobre aspectos vinculados al ambiente de trabajo, permite ser un aspecto diagnóstico que orienta acciones preventivas y correctivas necesarias para optimizar y/o fortalecer el funcionamiento de procesos y resultados organizacionales.

Con respecto a las variables que fue estudiado comportamiento organizacional para Carvajal, et al (2013), es a partir de la teoría del Campo 
de Lewin en 1951, donde se desarrolla por primera vez, al introducir el concepto de espacio vital, caracterizado por todos los hechos que determinan la conducta de un individuo en un momento determinado y que contiene todo aquello que le afecta, sea que esté o no dentro del espacio físico, y que es percibido de manera subjetiva. Respecto a la otra variable sostenida en la teoría de la expectativa, Alecoy (2012), propuso que las expectativas desarrolladas por las personas en el trabajo y la valoración que le conceden a determinados resultados ocupacionales, son variables motivacionales incidiendo en las preferencias, la persistencia del comportamiento laboral; así la expectativa factor de motivación para los empleadores siendo un indicador para recompensar el desempeño de manera proporcional ascendiendo a los mejores desempeños.

Frente a la realidad problemática se visitó a las bibliotecas especializadas de nuestro medio del mimo modo navegado por el internet, ubicando referentes o antecedentes; Castillo (2012) realizó la tesis titulada: Comportamiento organizacional en el campo educativo en Escuela Social de Avanzada, para optar el grado y magíster en educación, en la Universidad Rafael Urdaneta de Venezuela. Tuvo como objetivo, analizar el comportamiento organizacional del gerente educativo en la participación comunitaria de la escuela. Su estudio fue de tipo descriptivo, analítico y diseño no experimental, transaccional y utilizó un cuestionario que aplicó a una muestra comprendida por 100 sujetos, llegando a la siguiente conclusión: Se identificó los elementos el comportamiento organizacional: actitudes, personalidad, gerencia participativa, inteligencia emocional, habilidades sociales, conciencia emocional.

Cómo también; Quesada (2014) realizó la tesis, titulada: El liderazgo transaccional y el comportamiento organizacional en las instituciones educativas públicas "Ramón Castilla" y "Túpac Amaru" del distrito de comas, de la unidad de gestión educativa local $\mathrm{N}^{\circ} 04$, provincia y departamento de Lima, año 2012" en la Universidad Nacional Mayor de San Marcos, Lima, Perú. De tipo básico y diseño correlacional y utilizó cuestionarios para ambas variables que aplicó a una muestra comprendida por 112 docentes, llegando a la siguiente conclusión: El liderazgo transaccional se relaciona significativamente con el comportamiento organizacional en las instituciones educativas "Ramón Castilla" y “Túpac Amaru” del distrito de Comas, jurisdicción de la Unidad de Gestión Educativa Local $\mathrm{N}^{\circ} 04$. Esto significa que el 
comportamiento organizacional que se da mayoritariamente tiene que ver con el liderazgo transaccional que los directivos asumen regularmente en dichas instituciones educativas. Estos antecedentes del estudio son los más relevantes que contribuyeron a la investigación porque sus resultados coinciden con mi trabajo de investigación.

Los fundamentos teóricos indican que respecto al variable comportamiento organizacional según; Adam Smith en su postulado de su doctrina clásica afirma: "lo que se tiene como riqueza natural no la pierdas por tus actitudes desorganizadas". En sus parafraseo de Ascencio (2011), esta publicación presenta una "explicación sobre la calidad de organización que se debe a una sociedad justa y equitativa con un compromiso organizacional y la cultura optima del compromiso laboral" (p.12). Según este autor, Smith (2011) logró deducir que la división del trabajo no solo incrementa la productividad, sino también aumenta la capacidad y la práctica de cada trabajador, "al ahorrarse el tiempo que se pierde en cambiar de tareas y al fomentar la invención de equipo y maquinaria que no requirieran mano de obra" (p.12).Así mismo; Robbins y Judge (2013), mencionan que las organizaciones difieren entre sí en la calidad de los sistemas que desarrollan y mantienen, y en los resultados que alcanzan. La diversidad de resultados es producto sobre todo de modelos de comportamiento organizacional diferentes. Estos modelos constituyen el sistema de certeza que domina la manera de pensar de los directivos de una empresa y que, por lo tanto, afecta a sus acciones. Por consiguiente, es de suma importancia que los administradores adviertan la naturaleza, significación y efectividad de sus modelos, así como de los adoptados por quienes lo rodean.

Como también a la variable satisfacción laboral; Robbins (2014), la define como el conjunto de actitudes generales del individuo hacia su trabajo. Quien está muy satisfecho con su puesto tiene actitudes positivas hacia éste; quien está insatisfecho, muestra en cambio, actitudes negativas. Cuando la gente habla de las actitudes de los trabajadores casi siempre se refiere a la satisfacción laboral; de hecho, es habitual utilizar una u otra expresión indistintamente. Esta definición tiene la ventaja de considerar la satisfacción como un proceso aprendido, que se desarrolla a partir de la interrelación; Para efectos de esta tesis, se tomará en cuenta la definición propuesta por la psicóloga Sonia Palma Carrillo (1999), quien define la satisfacción laboral "como la predisposición frente al trabajo, basada en 
creencias y valores positivos, los cuales fueron generados por la rutina laboral." Tomando en cuenta factores como la significación de la tarea, condiciones laborales, reconocimiento personal/social y beneficios económicos (Palma, 2005, citado por Lozada, 2017).

Así mismo la investigación se realizó con el propósito de demostrar en qué medida el programa del comportamiento organizacional influye en la satisfacción laboral la cual resultó la influencia significativa esto contribuye al enriquecimiento del conocimiento científico en materia de mejorar y promover la cultura organizacional a través de un programa para una satisfacción laboral eficiente, permitiendo profundizar las teorías existentes.

Los problemas, objetivos e hipótesis formulados responden a; De qué manera el programa de comportamiento organizacional permite la satisfacción laboral en los docentes de la Carrera Profesional de Contabilidad de la Facultad de Ciencias Económicas y Administrativas de la Universidad Nacional de Ucayali, Pucallpa; 2019?, específicos; ¿De qué manera el programa de comportamiento organizacional permite la satisfacción del trabajo entre sí, en los docentes?,¿De qué manera el programa de comportamiento organizacional permite la satisfacción de sistemas con recompensas justas, en los docentes?,¡De qué manera el programa de comportamiento organizacional permite la satisfacción con las condiciones favorables del trabajo, en los docentes?,¿De qué manera el programa de comportamiento organizacional permite la satisfacción con la supervisión, en los docentes?, ¿De qué manera el programa de comportamiento organizacional permite la satisfacción con la compatibilidad y la personalidad en el puesto, en los docentes? Como objetivos de investigación fue: Analizar de qué manera el programa del comportamiento organizacional permite la satisfacción laboral en los docentes de la Carrera Profesional de Contabilidad de la Facultad de Ciencias Económicas, Administrativas y Contables de la Universidad Nacional de Ucayali, Pucallpa; 2019. Y específicos; Determinar de qué manera el programa del comportamiento organizacional permite la satisfacción del trabajo entre sí, en los docentes. Determinar de qué manera el programa del comportamiento organizacional permite la satisfacción de sistemas de recompensas justas, en los docentes. Determinar de qué manera el programa del comportamiento organizacional permite la satisfacción de las condiciones favorables del trabajo, en los docentes. Determinar de qué 
manera el programa del comportamiento organizacional permite la satisfacción con la supervisión, en los docentes. Determinar de qué manera el programa del comportamiento organizacional permite la satisfacción entre compatibilidad entre la personalidad y el puesto, en los docentes. Y las Hipótesis fueron: El programa del comportamiento organizacional permite significativamente la satisfacción laboral en los docentes de la Carrera Profesional de Contabilidad de la Facultad de Ciencias Económicas, Administrativas y Contables de la Universidad Nacional de Ucayali, Pucallpa; 2019. Y los específicos; El programa del comportamiento organizacional permite significativamente la satisfacción del trabajo entre sí, en los docentes. El programa del comportamiento organizacional permite significativamente la satisfacción de sistemas de recompensas justas, en los docentes; El programa del comportamiento organizacional permite significativamente la satisfacción de las condiciones favorables del trabajo, en los docentes ; El programa del comportamiento organizacional permite significativamente la satisfacción con la supervisión, en los docentes; El programa del comportamiento organizacional permite significativamente la satisfacción entre compatibilidad entre la personalidad y el puesto, en los docentes.

\section{Metodología}

Los materiales utilizados en la investigación fue el método científico basados en la manipulación de variables de estudio como instrumento cuestionario para medir la progresión de la aplicación del programa; fue un éxito a ello el estudio es de tipo experimental, en su nivel explicativo según el autor precisa; "conforme transcurre una la asociación causal de relación de las variables". (Sampieri, Collado \& Baptista, 2014). La investigación se ubicó en el diseño pre experimental; experimental porque se manipuló la variable independiente para ver sus efectos en la variable dependiente, tal como señala Sampieri, Collado \& Baptista (2014). "Lo que se hizo en la investigación experimental es observar fenómenos tal y como se dan en su contexto natural, para después analizarlos". Se trabajó con poblacional muestral de 18 docentes, con el muestreo no probabilístico denominado muestreo censal ya que los sujetos fueron los mismos de la población.

\section{Resultados y discusión}

Los resultados de acuerdo con los indicadores se evidencian que influye significativamente 
el programa de comportamiento dimensiones, como se percibe en la tabla y organizacional en la satisfacción laboral en los figura: docentes esto se evidencia inclusive entre sus

Tabla 1

Resultado del Programa de comportamiento organizacional y satisfacción laboral

\begin{tabular}{lcc|cc}
\hline & \multicolumn{2}{c|}{ Pre - Test } & \multicolumn{2}{c}{ Post - Test } \\
\cline { 2 - 5 } & $\mathbf{f i}$ & $\mathbf{h i \%}$ & $\mathbf{f i}$ & $\mathbf{h i \%}$ \\
\hline Nunca & 3 & $16 \%$ & 0 & $0 \%$ \\
Casi Nunca & 10 & $56 \%$ & 0 & $0 \%$ \\
A veces & 5 & $28 \%$ & 2 & $11 \%$ \\
Casi siempre & 0 & $0 \%$ & 1 & $6 \%$ \\
Siempre & 0 & $0 \%$ & 15 & $83 \%$ \\
Total & 18 & $100 \%$ & 18 & $100 \%$ \\
\hline
\end{tabular}

En la tabla y figura 1 ; se presenta los resultados esperados de la aplicación del programa de comportamiento organizacional en la satisfacción laboral en los docentes de la Carrera Profesional de Contabilidad de la Facultad de Ciencias Económicas, Administrativas y Contables de la Universidad Nacional de Ucayali-Pucallpa, 2019; los datos demuestran una diferencia muy significativa, en el pre test antes que se aplique el programa los resultados eran: Nunca (3), (16\%), Casi Nunca (10),( 56\%), A veces (5), (28), Casi siempre (0), (0\%), Siempre (0), (0\%). Estos datos evidencian que desconocían el compromiso organizacional para una satisfacción laboral mientras en el post test ya se nota la mejora después de aplicar el programa del compromiso organizacional arribando a los resultados; Nunca (0), (0\%), Casi Nunca (0), (0\%) A veces (2), (11\%) Casi siempre (1), (6\%) Siempre (15), (83\%). Es notable la comprensión que determina la significatividad por ello es recomendable que se debe promover el cumplimiento, mejora $\mathrm{y}$ productividad de los resultados esperados. 


\section{Resultado general}

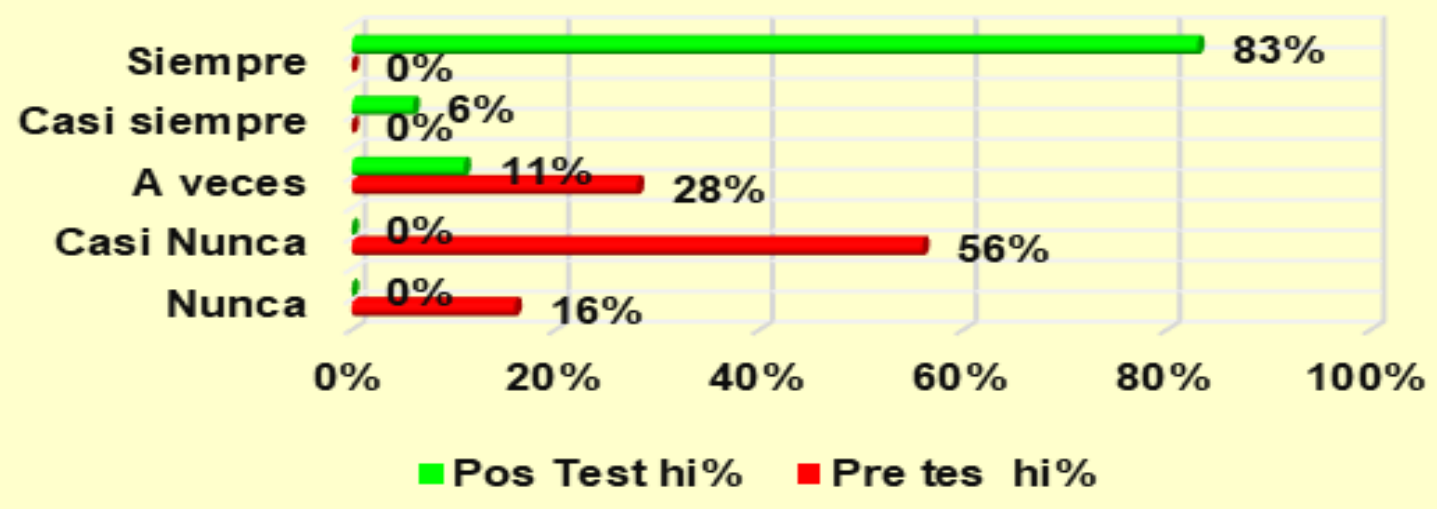

Figura 1: Resultado del Programa de comportamiento organizacional y satisfacción laboral

Se demuestra la determinación del programa para el comportamiento organizacional que permite la satisfacción del trabajo entre sí, en los docentes de la Carrera Profesional de Contabilidad de la Facultad de Ciencias Económicas, Administrativas y Contables de la Universidad Nacional de Ucayali, Pucallpa, 2019; se evidencia el logro significativo diferenciando del pre test con el post test. El porcentaje muy elevado es que desconocen que es importante el compromiso organizacional (67\%), aplicando el programa en el post test se revierte las cosas (83\%), demostrando la corroboración por su importancia y naturaleza de implementación con programas de formación y fortalecimiento de compromisos organizacionales.
Al determinar el programa del comportamiento organizacional que permite la satisfacción de sistemas de recompensas justas, en los docentes de la Carrera Profesional de Contabilidad de la Facultad de Ciencias Económicas, Administrativas y Contables de la Universidad Nacional de Ucayali, Pucallpa, 2019; donde el porcentaje es; $67 \%$ en el pre test respondiendo el nivel de desconocimiento más bajo, mientras en el post test después de la aplicación del programa demostraron que si es importante llegando al $78 \%$.

Del mismo modo al determinar que el programa del comportamiento organizacional permite la satisfacción de las condiciones favorables del trabajo, en los docentes de la Carrera Profesional de Contabilidad de la 590

Downloadable from: http://revistas.unu.edu.pe Carretera Federico Basadre Km 6, Dirección de Producción Intelectual

Revista de Investigación Universitaria por Universidad Nacional de Ucayali se distribuye bajo una Licencia Creative Commons Atribución-NoComercial 4.0 Internacional. 
Facultad de Ciencias Económicas, Administrativas y Contables de la Universidad Nacional de Ucayali, Pucallpa, 2019; llegando a un porcentaje de no comprensión $56 \%$ en el pre test, mientras en el post test, (44\%) Siempre (7), (39\%).

Es notable la comprensión que determina la significatividad del desempeño laboral depende de los compromisos organizacionales y buscar las condiciones favorables del trabajo supervisión. Así mismo finalmente se determinó que el programa del comportamiento organizacional permite la satisfacción entre compatibilidad entre la personalidad y el puesto, en los docentes de la Carrera Profesional de Contabilidad de la Facultad de Ciencias Económicas, Administrativas y Contables de la Universidad Nacional de Ucayali-Pucallpa, 2019.

Con respecto al planteamiento del problema; los resultados evidencian que fue un éxito implementar, ejecutar y evaluar el programa del comportamiento organizacional en la satisfacción laboral de los docentes del nivel superior sostenida los ideales en; los postulados del planteamiento del problema se demostraron que para la mejora continua del compromiso organizacional se debe aplicar un programa estratégico que integra y cubre las expectativas organizacionales como salariales y responsabilidades que coadyuve y ayude a una satisfacción laboral y mejorar los resultados de desempeño eficiente en los docentes del estudio propuesto. (Robbins, 2014, p.48). En relación con los antecedentes del estudio. En su estudio de Broomfield \&Thompson y Castillo (2016) Concluyen y señalan que no existen fórmulas simples y prácticas para trabajar con las personas, ni existe una solución ideal para los problemas de la organización. Todo lo que se puede hacer es incrementar la comprensión, y el análisis de las capacidades existentes para elevar el nivel de calidad de las relaciones humanas en el trabajo. Las metas son difíciles de alcanzar, pero poseen un gran valor, si se está dispuesto a pensar en las personas como seres humanos se puede trabajar eficazmente con ellas. Estos resultados cualitativos se evidencian y se contrasta con los resultados de mi investigación precisando que cuando se aplica un programa de comportamiento organizacional con evidencias operativas se mejora y aplicable para cambiar las actitudes de los empleados y docentes en especial en el estudio que fue propuesto es por ello se contrasta con los resultados de estudio que son similares, pero aclarando que no es una sola solución dependiendo de los compromisos de 
los recursos humanos. Así mismo Salazar, Mendoza y Jasso (2013), investigaron sobre Estructura organizacional y su relación con el desempeño donde los resultados estadísticos de este análisis evidencian que el tipo de diseño organizacional de las instituciones educativas de Hidalgo se caracteriza por ser predominantemente tradicional y conducen a un deficiente desempeño de los docentes. El $71 \%$ de las organizaciones con diseño innovador desarrolla plan estratégico, a diferencia de las empresas con diseño tradicional en las cuales únicamente el $40 \%$ lo realizan de manera parcial.

Precisamente para mejorar los bajos resultados como desempeño se coadyuva con la tesis de Salazar (2012) en promover la aplicación del programa de comportamiento organizacional para mejorar la satisfacción laboral como resultados desempeños eficientes y con mayor compromiso organizacional. En relación con la fundamentación teórica; Desde la filosofía de Robbins (2013) define al definir el comportamiento organizacional como un "campo de estudio que investiga el efecto que tienen los individuos, los grupos y la estructura sobre el comportamiento dentro de las organizaciones" (p.10). A este concepto, Hellriegel y Slocum (2009) Agregan que la efectividad de los individuos, los grupos y las organizaciones se ven influenciados por los procesos y las prácticas internas. Desde el sentido de comportamiento organizacional es poco funcional si bien es cierto implementado como un programa de fortalecimiento, motivación y desarrollo para lograr estándares de calidad en la satisfacción laboral como encontrar desempeños óptimos de los mismos, se precisa al demostrar que fue exitoso la aplicación del programa del comportamiento organizacional para rediseñar y generar una nueva concepción más sistémico del comportamiento organizacional. Estos resultados se evidencian en la tabla y figura 1; se presenta los resultados esperados de la aplicación del programa de comportamiento organizacional en le satisfacción laboral en los docentes de la Carrera Profesional de Contabilidad de la Facultad de Ciencias Económicas, Administrativas y Contables de la Universidad Nacional de Ucayali-Pucallpa, 2019; los datos demuestra una diferencia muy significativa, en el pre test antes que se aplique el programa los resultados eran: Nunca (3), (16\%), Casi Nunca (10),( 56\%), A veces (5), (28), Casi siempre (0), (0\%), Siempre (0), $(0 \%)$. Estos datos evidencian que desconocían el compromiso organizacional para una satisfacción laboral mientras en el post test ya se nota la mejora después de aplicar el 
programa del compromiso organizacional arribando a los resultados; Nunca (0), (0\%), Casi Nunca (0), (0\%) A veces (2), (11\%) Casi siempre (1), (6\%) Siempre (15), (83\%). Es notable la comprensión que determina la significatividad por ello es recomendable que se debe promover el cumplimiento, mejora y productividad los resultados esperados.

En relación con las hipótesis; se demuestra como el $\mathrm{t}$ calculado 9.633 es mayor que el $\mathrm{t}$ teórico o critico 2.048, y el P-valor es menor que 0.05 ; se rechaza la hipótesis nula y se acepta la hipótesis alterna, así mismo se corrobora que el objetivo formulado fue analizado de cómo el comportamiento organizacional permite la satisfacción laboral en los docentes de la Carrera Profesional de Contabilidad de la Facultad de Ciencias Económicas y Administrativas de la Universidad Nacional de Ucayali-Pucallpa, 2019, de tal manera que resultó óptimo después de su aplicación como programa.

\section{Conclusiones}

Se logró demostrar que el programa del comportamiento organizacional permite la satisfacción laboral en los docentes de la Carrera Profesional de Contabilidad de la Facultad de Ciencias Económicas, Administrativas y Contables de la Universidad
Nacional de Ucayali, Pucallpa; 2019 cuando se aplica como programa esto nos evidencia la tabla y figura 1 , y 05 ; demostrando en el pre test; $56 \%$ el resultado elevado mientras en el post test se afirma que el $83 \%$ existe mejora y significancia; por lo tanto permite de manera positiva su influencia y la manera de optimizar el comportamiento organizacional.

\section{Agradecimiento}

A los Doctores que impartieron su Sapiencia filosófica, cósmica y hermenéutica en la Administración Pública.

A mis colegas que compartieron su trayectoria, experticia de experiencia en las Entidades Públicas y Privadas.

Al Dr. Hermilio Trujillo Martínez, ilustre docente de la Universidad Nacional "Hermilio Valdizán" de Huánuco, un maestro y ejemplo a seguir en mi vida profesional, además para mí, un padre que Dios me puso en mi camino y hoy sigo sus pasos con mucho orgullo.

A mi asesor por cumplir el protocolo de consolidar la presente investigación.

\section{Referencia bibliográfica}

Arellano, R. (2017). Comportamiento del consumidor. México: Mc Graw Hill 
UNIVERSIDAD NACIONAL DE UCAYALI

Revista de Investigación Universitaria

Versión electrónica 2664 - 8423

ARTICULO ORIGINAL
Vol. $11 \mathrm{~N}^{\circ} 2$, pp. $583-594$, julio/diciembre 2021

Recibido 26/08/2021

Aceptado 11/10/2021

Publicado 30/12/2021
Castillo, M. (2012). Comportamiento organizacional en el campo educativo en Escuela Social de Avanzada Dr. Guillermo Quintero Luzardo de Zulia. Universidad Rafael Urdaneta de Venezuela

Cook, C. (2004). A meta-analysis of response rates in webor Internet-Based Surveys. Educational and PsychologicalMeasurement, 60(6), 821-836

Deming, E. (1991). Fuera de la crisis. Boston: Muralla.

Díaz, M. (2012). Factores del comportamiento organizacional que influyen en el desempeño de los docentes de las instituciones de educación superior. Universidad Autónoma de Querétaro de México

Drucker, P. (1998). La gerencia de empresas. Buenos Aires: Sudamericana

Elera, R. (2012). Gestión institucional y su relación con la calidad del servicio en una institución educativa pública de Callao. Universidad San Ignacio de Loyola, Lima, Perú.
Gibson, J. (2002). Las organizaciones: comportamiento, estructura, procesos. Santiago de Chile, Chile: Mc Graw-Hill Interamericana

Guerrero, G. (2012). Relación clima institucional y calidad educativa en las instituciones educativas estatales. Universidad Peruana La Unión, Lima, Perú.

Hernández, R.; Fernández, C. y Baptista, P (2010). Metodología de la investigación. México: Mc Graw-Hill.

Robbins, S. y Judge, T. (2013). Comportamiento organizacional. México: Pearson.

Salazar, A. (2012). Influencia del comportamiento organizacional en la satisfacción laboral. Universidad San juan Bautista de Chincha,

Salazar, B. Mendoza, J. y Jasso, S. (2013). Estructura organizacional y su relación con el desempeño: un análisis de las instituciones educativas. Universidad Autónoma de Hidalgo, México. 